\title{
Identidad personal en niños y adolescentes: estudio cualitativo
}

Flor Quiroga, Mg. ${ }^{a}$

Universidad de Chile, Chile

Claudia Capella, Ph.D. ${ }^{b}$

Universidad de Chile, Chile

Gabriela Sepúlveda, Ph.D ${ }^{c}$

Universidad de Chile

\author{
Bárbara Conca, Mg. ${ }^{d}$ \\ Universidad de Chile \\ Jenniffer Miranda, Ph.D. ${ }^{\mathrm{e}}$ \\ Universidad de Chile
}

fquiroga67@gmail.com

\section{Resumen (analítico)}

La construcción de identidad personal es un proceso evolutivo. Escasas investigaciones estudian este proceso en la infancia. El objetivo de esta investigación es describir la construcción de identidad personal de niños y adolescentes entre 6 y 18 años, desde un enfoque constructivista evolutivo. Se realiza un análisis temático de narrativas autobiográficas escritas por 119 niños y adolescentes. Los resultados se organizaron en torno a tres dimensiones de la identidad personal: unidad de identidad, integración de identidad, integración con otros; observándose que desde la infancia hay un reconocimiento personal, luego se integran diferentes eventos vitales significativos y en la adolescencia se complejiza su construcción, incorporando mayor reflexión y aspectos ideológicos. Se discuten implicancias para favorecer un desarrollo adaptativo de la identidad en todas las edades, validando diversidad de opciones.

\section{Palabras clave}

Identidad personal, imagen personal, narrativa, desarrollo del niño, niños, constructivismo, Piaget.

\section{Thesauro}

Biblioteca Dr. Jorge Villalobos Padilla, S. J. Iteso, Universidad Jesuita de Guadalajara.

\section{Para citar este artículo}

Quiroga, F., Capella, C., Sepúlveda, G., Conca, B., \& Miranda, J. (2021). Identidad personal en niños y adolescentes: estudio cualitativo. Revista Latinoamericana de Ciencias Sociales, Niñez y Juventud, 19(2), 1-25. https://dx.doi.org/10.11600/rlcsnj.19.2.4448

\section{Historial}

Recibido: 26.07 .2020

Aceptado: 28.01.2021

Publicado: 30.04 .2021

\section{Información artículo}

Este artículo se basa en la investigación realizada dentro del proyecto Semilla Construcción de identidad personal en niños y adolescentes de 6 a 18 años: aspectos evolutivos financiado por el Departamento de Psicología de la Universidad de Chile, entre Marzo 2014 y Julio 2017. Área: psicología. Se agradece al Liceo Manuel de Salas por la colaboración en este proyecto de investigación, así como a las alumnas colaboradoras del proyecto. 


\section{Personal identity in children and adolescents: a qualitative study}

Abstract (analytical)

Construction of personal identity is a developmental process. Few research investigates this process in children. The research objective is to describe the personal identity construction of children and adolescents between 6 and 18 years old, from a developmental constructivist approach. Thematic analysis of autobiographical narratives written by 119 children and adolescents is performed. Results were organized around the three personal identity dimensions: unity of self, integration of self and integration with others. Results show from childhood there is a personal recognition, then different significant life events are integrated, and in adolescense this construction becomes more complex, integrating more reflection and ideological aspects. Implications are discussed to promote an adaptive development of identity through lifespam, validating a diversity of options.

Keywords

Personal Identity, personal image, narrative, child development, children, constructivism, Piaget.

\section{Identidade pessoal em crianças e adolescentes: estudo qualitative}

Resumo (analítico)

A construção da identidade pessoal é um processo evolutivo. Poucas pesquisas investigam esse processo na infância. $O$ objetivo desta pesquisa é descrever a construção da identidade pessoal de crianças e adolescentes entre 6 e 18 anos, a partir de uma abordagem construtivista evolutiva. É realizada uma análise temática das narrativas autobiográficas escritas por 119 crianças e adolescentes. Os resultados foram organizados em torno de três dimensões da identidade pessoal: unidade de identidade, integração de identidade, integração com os outros; e mostram que desde a infância há um reconhecimento pessoal, logo diversos acontecimentos significativos da vida são integrados, e na adolescência sua construção torna-se mais complexa, incorporando mais reflexão e aspectos ideológicos. As implicações para promover o desenvolvimento adaptativo da identidade em todas as idades são discutidas, validando a diversidade de opções.

\section{Palavras-chave}

Identidade pessoal, si-mesmo, narrativas, desenvolvimento, crianças, Construtivismo, Piaget.

\section{Información autoras}

[a] Psicóloga. Magíster en Psicología, mención Psicología Clínica Infanto-Juvenil, Universidad de Chile. Académica Departamento de Psicología, Universidad de Chile. iD 0000-0003-4807-6904. H5: 1. Correo electrónico: fquiroga67@gmail.com

[b] Psicóloga. Master en Salud Mental Infanto-Juvenil. Doctora en Psicología, Universidad de Chile. Académica Departamento de Psicología, Universidad de Chile. (autora corresponsal). 0000-0002-7252-6605. H5: 5. Correo electrónico: ccapella@u.uchile.cl

[c] Psicóloga. Magíster en Educación, mención Psicología Educacional. Doctora en Filosofía mención Ética, Universidad de Chile. Profesora Titular, Universidad de Chile. iD 0000-0001-6646-4965. H5: 1. Correo electrónico: gsepulve@uchile.cl

[d] Psicóloga. Magíster en Psicología, mención Psicología Clínica Infanto-Juvenil, Universidad de Chile. (iD) 0000-0001-9607-3412. H5: 1. Correo electrónico: bconca@gmail.com

[e] Psicóloga. Magíster y Doctora en Psicología Clínica y de la Salud, Universitat Autònoma de Barcelona. Académica Departamento de Psicología, Universidad de Chile. iD 0000-0002-2565-3177. H5: 4. Correo electrónico: jennimiranda@gmail.com 


\section{Introducción}

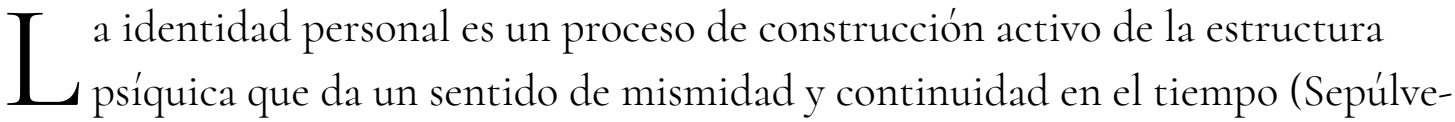
da, 2020). Esta construcción es un proceso evolutivo que se da durante toda la vida y está en permanente transformación (Erikson, 1993; Guidano, 1994; Sepúlveda, 2020). Diversos autores se han referido a la identidad (Ergün, 2020), existiendo diferentes perspectivas teóricas que coinciden en su constante desarrollo desde la infancia e incluso el nacimiento, otorgándole relevancia a este proceso en el desarrollo psicológico adaptativo (Beyers \& Luyckx, 2016; Negru-Subtirica et al., 2016; Oron \& Echarte, 2017).

No obstante, estos estudios se han enfocado principalmente en la adolescencia (Klimstra et al., 2010; Meeus, 1996; Zacarés et al., 2009), debido a lo cual, esta investigación pretende aportar nuevos conocimientos en la temática de identidad personal en niños ${ }^{1} \mathrm{y}$ adolescentes, describiendo la construcción de la identidad personal en niños y adolescentes de 6 a 18 años, utilizando un enfoque constructivista evolutivo.

Erikson es uno de los principales estudiosos de la identidad, integrando en este concepto el nexo entre individuo e historia, así como entre pasado y futuro. El concepto de identidad se refiere a «un proceso localizado en el centro del individuo, así como en el centro de su comunidad y cultura» (Erikson, 1994, p. 22). La identidad implica una sensación subjetiva de mismidad y continuidad, siendo un constante proceso de desarrollo hacia una progresiva diferenciación e inclusión (Erikson, 1993, 1994).

El concepto de identidad es central en el enfoque constructivista evolutivo, el cual pone el acento en la construcción de significados, a través de procesos de transformación y cambio, considerando variables evolutivas (Sepúlveda, 2020) y teniendo como referentes conceptuales principales a Jean Piaget y Jerome Bruner.

\footnotetext{
${ }^{1}$ Con «niños» nos referimos en el texto a niños y niñas, mientras que con «adolescentes» a las y los adolescentes, a no ser que se explicite el género.
} 
Desde este enfoque la identidad personal es un proceso evolutivo de diferenciación e integración, constituyéndose en un permanente movimiento de construcción del significado entre el sí mismo y el mundo, otorgando la posibilidad de ser reconocidos y reconocer a otros como seres únicos, inmersos en un contexto social y cultural (Sepúlveda, 2020). Kegan (1994) plantea que, a través de la vida, la persona va a desarrollar acciones para lograr la atención de otros, para reconocerse y ser reconocido, construyendo su identidad personal en su relación con los otros y con el mundo.

Siguiendo la línea constructivista, Bruner (1991) plantea la identidad personal como un constructo intersubjetivo, que permite el despliegue de una historia personal que se desarrolla, reflejando el proceso de construcción de significados que redefine al sí mismo en cada acción con los otros y con el mundo, dentro de una determinada cultura y tradición.

Desde un enfoque constructivista evolutivo, Sepúlveda (2020), integrando distintos referentes teóricos, plantea tres procesos en la organización de la identidad personal: unidad de la identidad, integración de la identidad e integración con otros. La unidad de la identidad implica la afirmación del sí mismo, el reconocimiento de sí como ser único y diferente, respondiendo a la pregunta ¿quién soy?; ello a través de un proceso de diferenciación psicológica, corporal y sexual, con el reconocimiento de características personales, valores e ideologías que configuran el carácter y, a la vez, la tarea de diferenciación implica el reconocimiento del otro como semejante a sí mismo y constituyente del sí mismo. Es una tarea de interpretación del sí mismo, que lleva a la estima y el respeto de sí, haciéndose consciente y responsable de sus actos. Implica la toma de conciencia de las limitaciones propias e inherentes a la condición humana, así como de la igualdad, reconociendo la libertad propia y del otro en la esfera de la acción (Sepúlveda, 2020).

La integración de la identidad es una tarea de acomodación a las experiencias que se han dado en la historia vital. Implica la integración del presente, pasado y futuro acontecido en la trayectoria vital del sujeto a modo de experiencias personales significativas, lo que da un sentido de continuidad al sí mismo. Requiere una integración de roles con una perspectiva histórica y la búsqueda activa en el presente para ampliar el campo experiencial a través de la acción, definiendo y descubriendo principios y motivos, los que darán el sentido de consistencia en el tiempo a la unidad de sí (Sepúlveda, 2020).

La integración con otros implica la búsqueda de alternativas de acción y de reconocimiento desde otros, a través de la incorporación a grupos de reflexión y de acción. La integración a grupos da la posibilidad de actuar en el mundo, de poner su definición de identidad al servicio de los otros, según sus posibilidades de realización (Sepúlveda, 2020). 
A nivel internacional, escasas investigaciones se centran en la comprensión de los aspectos evolutivos de la identidad personal y en los momentos de cambio que se dan en ella, focalizándose más bien en la etapa adolescente (Klimstra et al., 2010; Meeus, 1996; Zacarés et al., 2009) o en los aspectos psicopatológicos o traumáticos de la identidad (Beyers \& Luyckx, 2016; Capella, 2017; Foelsch et al., 2015; Kirk \& Hinton, 2019). En el caso de niños y adolescentes, los estudios se centran principalmente en el autoconcepto y la autoestima, especialmente en niños (Tatlow-Golden \& Guerin, 2010), en la identidad racial, nacional o de género (Murphy, 2018; Rivas-Drake et al., 2017; Turban \& Ehrensaft, 2018) o en instrumentos y metodologías de evaluación de la identidad personal (Crafter et al., 2015; Foelsch et al., 2015; Goth et al., 2012; Klym \& Cieciuch, 2015; Procaccia et al., 2014; Thomas, 2015, Valdés et al., 2019). En Latinoamérica se han realizado trabajos con metodología cualitativa con adolescentes y jóvenes respecto a aspectos culturales, étnicos o raciales en la construcción de identidad o construcción de identidades alternativas en poblaciones específicas (Cifuentes-Muñoz \& Rojas-Jara, 2018; Díaz-Barriga et al., 2019; Mansilla \& Piñones-Rivera, 2017; Ortiz-Piedrahía, 2020).

Desde el enfoque constructivista evolutivo, en Chile se han desarrollado algunos estudios con niños y adolescentes, con muestras principalmente clínicas, enfocándose en aspectos de la identidad personal como el desarrollo de sí mismo (Catalán, 2004; De la Harpe, 2005; Martin, 2000), su relación con la psicopatología (Crockett, 2013; González, 2011; Martin, 2000; Ramírez \& Salas, 2013) y en grupos o comunidades específicas (Capella, 2017; Hernández, 2012; Rossel \& Torrejón, 2019; Yáñez, 2014).

El desarrollo evolutivo de la identidad personal, especialmente desde el enfoque constructivista evolutivo, es un desafío pendiente en la investigación a nivel nacional e internacional, siendo necesario conocer cómo se construye la identidad personal en la infancia y adolescencia para promover su desarrollo adaptativo, a través de intervenciones clínicas, psicoterapéuticas y preventivas. Así, la pregunta que guía la presente investigación es: ¿cuál es la construcción de identidad personal en niños y adolescentes entre 6 y 18 años?

Se utilizará un enfoque cualitativo que permita, a través de las narrativas personales de los mismos niños y adolescentes, acercarse a esta construcción, buscando ser un aporte novedoso al conocimiento de este tema a nivel internacional, al incorporar el estudio de la identidad, no solo en adolescentes, sino también en niños, más allá de un enfoque de autoconcepto o identificaciones en la infancia, como comúnmente se ha realizado. Por otro lado, resulta novedoso estudiar empíricamente estos constructos teóricos en 
Chile, siendo un aporte el estudio desde el enfoque constructivista evolutivo, debido al énfasis de esta teoría en los procesos identitarios y evolutivos en la infancia y adolescencia.

\section{Método}

\section{Diseño y muestra}

Esta investigación utiliza un método cualitativo, coherente con la epistemología constructivista que guía este estudio, ya que se busca comprender e interpretar la realidad tal y cómo es significada por los participantes a partir de sus experiencias subjetivas (Rodríguez et al., 1999); así mismo, es descriptiva, ya que intenta caracterizar el fenómeno estudiado (Hernández et al., 2006).

La selección de la muestra fue de carácter intencionado. Se seleccionaron aquellos casos que cumplieran con los criterios de inclusión (niños y adolescentes entre 6 y 18 años, escolarizados, de la ciudad de Santiago, Región Metropolitana, Chile). Se seleccionó un colegio particular de nivel socioeconómico medio, con el fin de homogeneizar la variable y acceder a una muestra más representativa de ella —nivel socioeconómico medio representa al 57.4\% de la población nacional— (Asociación de Investigadores de Mercado, 2015).

Como el propósito del estudio era analizar la identidad personal en niños y adolescentes que presentaran un equilibrio adaptativo, se utilizó un cuestionario de tamizaje de psicopatología: el cuestionario de fortalezas y debilidades (Goodman, 1997), en su versión para padres, adaptado a Chile (Brown et al., 2014). A partir de este tamizaje se excluyeron de la muestra cinco participantes (dos hombres y tres mujeres) que presentaron algún tipo de psicopatología en el SDQ.

La muestra final quedó constituida por 119 casos, 72 mujeres y 47 hombres. Considerando el desarrollo psicológico se establecieron dos rangos etarios: de 6 a 11 años (infancia) y de 12 a 18 años (adolescencia) (Papalia et al., 2010) (tabla 1).

\section{Instrumentos}

Las autobiografías son documentos personales, considerados dentro de las técnicas biográficas en investigación cualitativa en ciencias sociales, siendo coherentes con la evaluación constructivista (Capella, 2013). 
Tabla 1

Muestra de participantes según rango de edad y sexo

\begin{tabular}{lccc}
\hline \multicolumn{1}{c}{ Rango edad } & Mujeres & Hombres & Total por rangos \\
\hline Infancia: 6-11 años & 34 & 20 & 54 \\
Adolescencia: 12-18 & 38 & 27 & 65 \\
Total & 72 & 47 & 119 \\
\hline
\end{tabular}

Se utilizó el formato planteado por Sepúlveda (2007, como es citado por Capella, 2011) con leves modificaciones por Capella (2011), en el cual se solicita a los niños y adolescentes la redacción de una autobiografía con estilo libre a partir de la consigna: «Escribe una historia sobre ti y tu vida, pudiendo incluir aspectos tales como, por ejemplo, una presentación personal en cuanto a quién eres, cuáles han sido los hechos o situaciones más importantes de tu vida, cómo imaginas tu futuro, entre otros».

\section{Procedimiento}

Esta investigación fue aprobada por el Comité de Ética de la Investigación de la Facultad de Ciencias Sociales de la Universidad de Chile. Se contactó a las autoridades y coordinadores del colegio, explicándoles la investigación y recibiendo la autorización respectiva. Luego, se seleccionaron al azar los cursos que participarían y se estableció contacto con los profesores jefes. En reuniones de padres en el colegio se presentó la investigación, incluyendo los aspectos éticos como la voluntariedad y confidencialidad y se respondieron consultas. A los padres que accedieron a participar se les solicitó la firma de un consentimiento informado, rellenar una ficha con datos básicos del niño y completar el SDQ para padres. Luego, durante la jornada escolar, se contactó a los niños y adolescentes de los cursos seleccionados explicándoles la investigación; aquellos interesados en participar firmaron asentimiento informado. Solo participaron aquellos niños y adolescentes que tanto ellos como sus padres aceptaron. Finalmente, en una sala del colegio en presencia de las investigadoras, los participantes —en grupos de no más de 4realizaron la autobiografía escrita de manera libre y sin tiempo límite.

\section{Análisis}

Las autobiografías se analizaron a través de la técnica de análisis temático teórico (Braun \& Clarke, 2006), realizándose un análisis de los temas emergentes en las autobiografías, focalizado en aspectos relevantes para la investigación, basados en el modelo teórico, 
siendo un tipo de análisis más inductivo. Este análisis se ajusta a las necesidades epistemológicas y metodológicas de la investigación, siendo coherente con el enfoque constructivista utilizado.

Cada autobiografía se analizó individualmente, realizándose un proceso analítico de identificación de los principales temas observados. Luego se realizó un análisis entre casos, agrupando los temas identificados en esquemas de clasificación cada vez más abarcadores. Acorde a metodologías de análisis cualitativas, se realizó durante todo el proceso el método de comparación constante, para el establecimiento de los análisis individuales de las autobiografías y la creación de temas comunes entre los casos (Braun \& Clarke, 2006). La comparación constante alude al contraste que se realiza en el proceso de análisis de los datos para la construcción de códigos y categorías de análisis de forma simultánea, comparando sus características similares y diferentes, así como contrastando con elementos teóricos (Bonilla-García \& Lopez-Suárez, 2016). Se desarrollaron temas de carácter emergente relativos a la identidad personal, que se organizaron en torno a las dimensiones de la identidad personal planteadas teóricamente (Sepúlveda, 2020). También se realizaron análisis intercasos diferenciados por rangos etarios.

Para asegurar la validez de los resultados, se empleó la estrategia de triangulación (Cornejo \& Salas, 2011), comúnmente utilizada como estrategia en investigación cualitativa, en que diversos investigadores participaron en los procesos de análisis de los datos, siendo validados a través de un acuerdo intersubjetivo.

\section{Resultados}

Los resultados se presentan según rangos etarios de infancia y adolescencia, así como dimensiones de la identidad personal, destacando aspectos diferenciales relevantes dentro de cada rango etario.

\section{Identidad personal en la infancia}

\section{Unidad del sí mismo}

Los niños se reconocen a sí mismos principalmente a partir de datos personales (nombre, edad), demográficos (lugar de nacimiento y residencia, cambios de comuna, ciudad o país) y escolares (curso, colegio). Solo algunos se describen a partir de características físicas y psicológicas. 
Yo soy (nombre) y tengo 8 años y tengo 2 ojos y son de color café y me gusta jugar. (Niño, 8 años)

Yo a veces soy pesada y la mayoría de las veces soy simpática. (Niña, 9 años)

En algunos casos, el reconocimiento de sí mismos incorpora el reconocimiento de otros.

Todos dicen que soy linda y divertida. (Niña, 9 años)

Todos ellos [padres y hermano] me ven como una niña buena y a la vez inteligente y hermosa. (Niña, 10 años)

La mayoría de los niños se reconocen a sí mismos por sus intereses y gustos, destacando lo lúdico (videojuegos, parque de diversiones), deportivo (fútbol, bicicleta) y alimentario (especialmente en las autobiografías de niños de 6 a 8 años). El interés por animales y mascotas aparece a partir de los 9 años. En menor medida se observan las festividades preferidas como cumpleaños y los intereses académicos (asignaturas favoritas):

Me gusta comer lechuga. Me gustan los animales y la istoria ${ }^{2}$ y me gustan las plantas. Me gustan los cumpleaños y además lenguaje. (Niño, 6 años)

Tambien me gustan mucho los animales, la música me facina, me encanta cantar. (Niña, 11 años)

Algunos niños se reconocen y valoran a sí mismos a partir de actividades en las cuales sienten que tienen habilidades, en lo académico, artístico e interpersonal.

A veces siento que soy la mejor amiga de todas las de mi curso y algunos hombres de mi curso. (Niña, 8 años)

Soy una buena alumna, soy inteligente, me gusta el arte, soy buena haciendo calculos y soy buena escaladora. (Niña, 9 años)

\section{Integración del sí mismo}

Las autobiografías aluden a la temporalidad, integrando el pasado, presente y futuro en los relatos personales. Desde el pasado, a partir de los 9 años, los niños mencionan eventos significativos en su historia vital, integrando hechos específicos (inicio de su etapa escolar, cumpleaños) y momentos de cambios evolutivos, dando continuidad a su historia vital. Describen además sus reacciones emocionales o afectivas, destacando sentimientos

\footnotetext{
${ }^{2}$ Las narrativas están presentadas verbatim, sin corregir los errores cometidos.
} 
de tristeza, superación, disfrute o entusiasmo por haber superado situaciones difíciles. Esta descripción de eventos se presenta escasamente en niños menores de esa edad.

Nací (fecha), apenas entré al jardín empezé a tener amigos; a los 3 años aprendí a leer y escribir, al siguiente año entré al colegio, cuando cursaba 1ero básico empezé a tener gustos más definidos respecto a la música, ropa, etc. (Niño, 11 años)

Dentro de los eventos relevantes en lo familiar destacan las separaciones conyugales de los padres, enfermedades de miembros de la familia (incluyendo mascotas), así como la incorporación y pérdida de figuras significativas por fallecimientos.

Yo estaba en la casa de mis abuelos y mi abuelo estaba enfermo estaba jugando con mi hermana y mi prima cuando nos llaman yo fui a la pieza de mi abuelo y estaba dormido y mi mamá estaba llorando yo no sabía que hacer. (Niño, 9 años)

Yo con mi familia me llevo muy bien, el año pasado mis papás se separaron y a mi me dio pena. (Niña, 10 años)

En el ámbito interpersonal, mencionan el momento en que inician una amistad con algún compañero.

Una de las situaciones mas importantes de mi vida fue conocer a mis amigas porque ellas me han apollado en todo, siempre estan hay en las buenas y en las malas, ellas han sido un cambio inportante en mi vida y sin ellas no ubiese logrado muchas cosas. (Niña, 11 años)

En su historia escolar mencionan el inicio de la escolaridad y de actividades tanto deportivas como artísticas. Otros eventos que destacan son los cambios de domicilio o colegio.

Entré al colegio en kinder y ahora estoy en $4^{\circ}$ básico, y me e hecho muchas amigas y las quiero mucho. (Niña, 9 años)

Respecto del presente los niños refieren situaciones que son relevantes para ellos en lo familiar, social o escolar. Algunos niños expresan su satisfacción con la vida al momento de elaborar su autobiografía.

Abeses me peleo con mi ermano. juego con mis amigos. (Niña, 6 años)

Tengo una muy buena vida mi padre y mi madre me tratan como si fuera un diamante tambien tengo una hermanita que es mi angelito en silla de ruedas tengo un hermano que 
el si me trata como un diamante y mi tia (nombre) ella es la persona que ilumina mi vida... (Niña, 10 años)

PD. No me gusta nadie, solo encuentro guapo a un niño de $6^{\circ}$. (Niña, 11 años)

Hacia el futuro, desde los 9 años se observan proyecciones en diversos ámbitos, siendo la más frecuente la profesional, destacando deseos de éxito y bienestar (personal, social y económico). En muchos casos la proyección a futuro se relaciona con intereses actuales.

Yo quiero que este sea mi Futuro: yo quiero ser veterinaria porque amo a los animales, quiero viajar mucho porque me gusta y quiero tener una familia feliz. (Niña, 1o años)

Me gusta jugar fútbol (...) mi futuro lo veo que quiero ser futbolista. (Niño, 10 años)

Yo quiero una historia inigualable, mi sueño es ser el mejor mago del mundo entre otros

(...), quiero una vida buena. (Niño, 11 años)

Me imagino exitoso, pero bueno, eso depende de mí, ¿ No? (Niño, 11 años)

En algunos casos también aparecen proyecciones vinculadas al plano afectivo y social.

Me imagino en el futuro como una buena persona, y espero tener los mismos amigos que ahora. (Niña, 10 años)

\section{Integración con otros}

Los niños destacan y valoran sus relaciones y actividades con la familia nuclear y extensa. Describen la configuración familiar y con quiénes viven (incluyendo mascotas).

Micosa mas importante es mi mamá y mi papá. (Niña, 7 años)

(...) entonces yo casi todas las vacaciones voy a Suiza, y a mi me encanta porque puedo aprender el idioma, comer cosas ricas, y lo mejor es que tengo mis abuelos y mis primas favoritas ahí (...). Yo creo que toda mi familia es muy buena. (Niña, 10 años)

En el ámbito escolar, es frecuente que mencionen quiénes son sus amigos y la valoración que hacen de estos, así como la pertenencia al colegio.

Mis mejores amigas son (nombres). (Niña, 9 años)

(...) y mis compañeros de la vida los amo son muy importantes en mi vida y amo también mi colegio (...) que me puede entregar una mejor educación. (Niña, 10 años) 
Excepcionalmente algunos describen vivencias insatisfactorias con sus pares al haber sido víctimas de bullying.

A veces me siento sola porque mis compañeras no me toman en cuenta y me dejan aparte (...) Tengo una compañera que me molesta me mira con cara de desprecio y me molesta y yo me siento mal. (Niña, 8 años)

Cuando iba en 4to Algunos niños me icieron buling me desian gorda y gracias a eso tuve que ir al psicologo por 1 año. (Niña, 11 años)

Fui a un colegio que se llama (nombre) estuve 5 años en ese colegio me tuve que ir porque mis compañeros me trataban mal. (Niño, 11 años)

Mencionan la integración a grupos de pertenencia, principalmente relativos al colegio, como grupos scout o equipos deportivos.

Las cosas más importantes que me an pasado Ej. Cuando entre al club a jugar futbol, cuando ataje mi primer penal, cuando entre a este colegio, cuando ise mi primer amigo. (Niño, 11 años)

\section{Identidad personal en la adolescencia}

Los adolescentes se describen a sí mismos en las diferentes dimensiones de la identidad, observándose autobiografías con mayor descripción de contenidos.

\section{Unidad del sí mismo}

Los adolescentes, al igual que los niños, se describen a sí mismos a través de su identificación personal (nombre, edad, datos de nacimiento), aspectos demográficos (lugar de origen) y escolares (colegio o curso). Los adolescentes entre 12 y 15 años incluyen el sexo como parte de dicha identificación.

Me llamo (nombre) tengo 13 años, soy chilena francesa. (Mujer, 13 años)

Yo soy una chica de 14 años. (Mujer, 14 años)

El reconocimiento personal integra diversos aspectos personales, físicos, emocionales y sociales.

Me importa lo que piensan de mí y también que las personas me tengan confianza. (Hombre, 14 años) 
Soy una niña tímida, me gusta mucho sonreír y soy estudiosa. (Mujer, 13 años)

Estoy segura que soy una mujer fuerte, creativa, ágil, emocional y critica, tan segura como que me llamo J. (Mujer, 18 años)

Al igual que los niños, también se valoran y describen a través de intereses y actividades (académicas, artísticas, deportivas y musicales).

Me gusta cantar y gané $2^{\underline{0}}$ y $3^{\underline{o}}$ lugar en el festival de la voz del colegio. Hago teatro y realmente me gusta. Me encanta la música y la lectura ambos son una de las mejores cosas que me han pasado. Amo leer, encuentro que es una de las cosas mas maravillosas. (Mujer, 14 años)

Me metí a esgrima y sali $5^{\underline{0}}$ mejor a nivel nacional, quizás, mi mayor logro en 4 años. (Hombre, 14 años)

Un aspecto que aparece en los adolescentes, y que se acentúa desde los 15 años en adelante, es la integración de aspectos ideológicos en el ámbito social, religioso, político, entre otros, que son importantes en la caracterización personal.

Estos 2 últimos años he leído a muchos teóricos de tinte libertarino, definiéndome (pero sin curarme) como anarquista. (Hombre 15 años)

Hace dos años que milito en las juventudes comunistas de Chile y han sido importantísimos para mi, para mi pensamiento y mi forma de actuar. También soy gracias a mis padres, quienes me han apoyado y me inculcaron que el amor hacia los demás y la búsqueda de justicia son lo mas importante en esta vida. (Hombre, 17 años)

En los adolescentes aparecen deseos de independencia y autonomía, que se relacionan con la necesidad de toma de decisión y reafirmación de la agencia personal.

Como dije antes, lo único que quiero ahora es poder ser yo misma en pleno, sin restricciones. (Mujer, 16 años)

Soy vegetariana hace un año dos meses (...) Prefiero un poco más de libertad, ya que tampoco tengo demasiado tiempo para comprometerme. (Mujer, 17 años)

\section{Integración del sí mismo}

Desde el pasado, los adolescentes integran diversos eventos visualizados por ellos mismos como relevantes; incluso desde los 15 años integran eventos ocurridos antes de 
su nacimiento, enfatizando cambios vitales, tales como el reconocimiento de la orientación sexual.

Por distintos factores mis padres chilenos se enamoraron y conocieron en Francia, donde vivieron sus primeros años de matrimonio y me tuvieron a mí junto con mi hermana. (Hombre, 17 años)

En segundo medio asumí abiertamente mi homosexualidad, hecho que quizás sea el más importante de mi vida. (Hombre, 17 años)

Destacan cambios familiares (separación conyugal de sus padres, nuevas configuraciones familiares, nacimientos, fallecimientos) y sentimientos asociados a ellos, que oscilan desde tristeza o dolor, a no sentirse afectados emocionalmente.

Viví con mi padre hasta los 9 años y luego decidió marcharse a España. Lo he visto un par de veces y aunque yo no quiera, me afecta hasta el día de hoy su ausencia. (Mujer, 15 años)

También mencionan hechos que para ellos resultan relevantes en el ámbito escolar, tales como cambio de colegio e inicio de la escolaridad. En otros casos refieren cambios habitacionales (vivienda/comuna/ciudad/país).

Creo que uno de los hechos importantes y que más marcó mi vida fue cuando tuve que ir a vivir a España, tenía 9 años. (Mujer, 14 años)

A mis 6 meses de vida me vine a vivir a Santiago junto con mi familia en busca de oportunidades en el país natal de mis papás. (Hombre, 17 años)

En el presente mencionan reflexiones, deseos y anhelos actuales.

Bueno, ya me harté, soy una persona que quiere disfrutar la vida. (Mujer, 14 años)

Hoy veo el amor como algo muy ajeno a mí que no forma parte de mis intereses. (Mujer, 18 años)

Es frecuente que aparezca una evaluación de la vida o de la etapa vital en que se encuentran, integrando las diferentes experiencias vividas.

Los errores, problemas, dificultades te ayudan, aunque en el momento sientas rabia, impotencia o incluso nunca los llegues a entender y hasta el día de hoy sientes pena o rabia, en el fondo te ayudan a crecer y hacerte más fuerte. (Mujer, 14 años) 
Pero a pesar de todo, siento que tuve una infancia muy amena, y me siento muy afortunada con la vida que me han entregado los distintos personajes que me rodean, porque la han hecho muy feliz. (Mujer, 17 años)

En el futuro aparece más frecuentemente una proyección en diferentes ámbitos, primando los deseos en el ámbito profesional/laboral, incluyendo proyecciones laborales en lo deportivo y artístico, aspirando a trabajar en algo que les guste.

También yo quiero llegar a ser un gran jugador de hockey, ser campeón mundial, además nunca olvidar el equipo donde empecé. (Hombre, 13 años)

Me imagino un futuro con muchas oportunidades, haciendo lo que me gusta, y a pesar que desde muy chica me interesa la medicina, nadie sabe lo que nos depara el futuro... (Mujer, 15 años)

También se observan proyecciones en los ámbitos personal, familiar e interpersonal. Excepcionalmente, aparecen opciones que incluyen la solidaridad.

Estudiaré algo relacionado con medicina y así podré ayudar a la gente en temas de salud, así también podré encargarme de cuidar muy bien a mi familia y a mis seres queridos.

(Mujer, 16 años)

Para el futuro, anhelo la felicidad y la paz constante, pienso estudiar en la universidad y tener familia al igual como lo hizo mi mamá, un gran ejemplo para mí. (Mujer, 18 años)

Entre los 12 y 15 años, disminuye la mención a proyecciones futuras y, en algunos casos en que aparece, se observa una proyección a futuro poco clara y definida.

No me imagino mi futuro. (Hombre, 12 años)

No sabría que hacer en el futuro pero creo que es algo que viene que se hace en el tiempo que no es necesario proponerla ni ordenarla, solo pasa. (Mujer, 13 años)

\section{Integración con otros}

Los adolescentes se reconocen a sí mismos mediante relaciones y actividades con familiares significativos, valorando el tipo de relación establecida.

Un hecho importante en mi vida ha sido la muerte de un ser querido, mi bisabuela con la cual pasaba mucho tiempo ya que vivíamos en el mismo edificio (...). Era maravillosa, dudo que algún día yo pueda conocer a alguien que se asemeje un poco a ella (...). Ella hizo que yo me convirtiera en la persona que soy ahora. (Mujer, 14 años) 
Con mi padre siempre andamos en bicicleta o vamos a la feria, con mi madre somos totalmente compinches (...). Mis abuelos siempre han sido factores importantes de mi vida, muchas de las enseñanzas provienen de ellos, todos muy cultos y grandes personas, al igual que mis padres y hermanos. (Mujer, 18 años)

En lo escolar, algunos adolescentes se reconocen desde la pertenencia al colegio y su grado de integración social. Es frecuente que describan el tipo y calidad de las relaciones establecidas con sus pares, mencionando sentimientos de integración, apoyo de los amigos o sentimientos de exclusión y dificultad en entablar amistades. Esto aparece en algunos casos entre los 12 y 15 años.

(...) y por último, cambiarme a este colegio fue muy importante, por que desarrolle mis intereses y me siento muy integrada. (Mujer, 13 años)

He tenido una básica difícil, sin amigos, con mal carácter, golosa (en el sentido de tener ganas de tener y tener y tener más amigos). (Mujer, 13 años)

Me gusta mucho mi colegio por que tengo muchos amigos que me ayudan en todo. (Mujer, 13 años)

En relación a la integración con grupos de pertenencia, en algunos casos aparece principalmente en relación con el colegio, grupos de scout, equipos deportivos, entre otros.

Es así como desde pequeña he sido scout. (Mujer, 18 años)

\section{Discusión}

El análisis realizado de las autobiografías permitió identificar los tres procesos de la identidad personal en niños y adolescentes, de manera concordante con los aspectos teóricos desde el constructivismo evolutivo (Sepúlveda, 2020). Otro aspecto central es que las narrativas reflejan el desarrollo psicológico, evidenciándose en las distintas edades características del desarrollo evolutivo, descritas por Piaget (1995) y observándose elementos comunes y diferenciados de la identidad según las diferentes etapas etarias (figura 1).

En relación a las distintas dimensiones de la identidad, en la unidad del sí mismo los niños y adolescentes se describen y reconocen como seres únicos y diferentes a través de aspectos concretos, como características físicas y psicológicas, gustos, intereses y actividades desarrolladas, especialmente en aquellas en donde sienten tener habilidades. 
Figura 1

Principales resultados de la identidad

\begin{tabular}{|c|c|c|c|}
\hline $6-8$ & $9-11$ & $12-15$ & $16-18$ años \\
\hline \multicolumn{4}{|c|}{$\begin{array}{l}\text { Elementos comunes a todas las edades: } \\
\text { conocimiento personal centrado en datos personales, intereses y gustos. } \\
\text { valoración y validación del sí mismo es el logro alcanzado en rendimientos y habilidades en } \\
\text { diferentes ámbitos de acción. } \\
\text { e integración de las relaciones familiares y con amigos en la identidad personal. }\end{array}$} \\
\hline $\begin{array}{l}\text { - Reconocimiento personal, a } \\
\text { partir de aspectos tangibles } \\
\text { y sensoriales de la realidad. }\end{array}$ & $\begin{array}{l}\text { - Se comienza a incorporar } \\
\text { la integración del pasado, } \\
\text { presente y futuro a través } \\
\text { de la narración y reflexión } \\
\text { de eventos significativos y } \\
\text { también a partir de la } \\
\text { proyección a futuro en el } \\
\text { ámbito profesional. }\end{array}$ & $\begin{array}{l}\text { - Comienzan a integrar } \\
\text { diferentes características } \\
\text { personales psicológicas, } \\
\text { incluyendo el sexo en la } \\
\text { descripción de si mismos. } \\
\text { - La proyección hacia el } \\
\text { futuro, en algunos casos, } \\
\text { es poco definida y } \\
\text { confusa. } \\
\text { - Aparece una mayor } \\
\text { integración de las } \\
\text { relaciones establecidas } \\
\text { con figuras significativas. } \\
\text { Algunos casos mencionan } \\
\text { dificultades con pares } \\
\text { como temática relevante. }\end{array}$ & $\begin{array}{l}\text { - Se comienzan a integrar } \\
\text { aspectos ideológicos en la } \\
\text { descripción del si mismo. } \\
\text { - Integran y se complejiza la } \\
\text { reflexión en torno a eventos } \\
\text { vitales, incluyendo eventos } \\
\text { significativos previos al } \\
\text { nacimiento. } \\
\text { - La proyección hacia el } \\
\text { futuro no sólo se da en lo } \\
\text { laboral/profesional, sino } \\
\text { también en términos de } \\
\text { bienestar personal y de } \\
\text { construir familia propia. }\end{array}$ \\
\hline
\end{tabular}

Dado que el proceso de diferenciación implica la estima y respeto de sí y del otro que me reconoce y que es reconocido por cada persona en un plano de igualdad, reconociendo la libertad propia y del otro en la esfera de la acción (Sepúlveda, 2020), el apoyo a este proceso de diferenciación implica el reconocimiento de las necesidades, intereses y habilidades específicas de cada persona, estimulando la valoración de lo distinto, y no, en palabras de Byun-Chul Han (2017), la expulsión de lo distinto, que es lo que permite el descubrimiento de la propia identidad y el sentido de vida. Esto implica la necesidad del reconocimiento y valoración de lo completamente distinto que me permite a la vez reconocerme a mí mismo.

En todas las etapas del desarrollo incluidas en este estudio se observa que un elemento esencial en la valoración y validación del sí mismo es el logro alcanzado en rendimientos y habilidades en diferentes ámbitos de acción, según la valoración externa a nivel escolar, familiar o social, destacando el rendimiento en ámbitos escolares en esta valoración. En otro estudio realizado en Chile, Chávez y Vergara (2017), reportan desde la perspectiva de niños respecto a la escuela, una experiencia de trabajo, agobio y pérdida de libertad para realizar actividades recreativas, centrando el aprendizaje en la obtención de un buen rendimiento. 
En la integración del sí mismo se observa el sentido de continuidad en el tiempo, tanto en niños como adolescentes, con narrativas que integran eventos pasados, presentes y proyecciones futuras. Como eventos relevantes del pasado mencionan los cambios en la configuración familiar, social y escolar, relacionados con incorporaciones o pérdidas de vínculos afectivos. Entre estos destaca la separación de los padres como un evento significativo en sus vidas, lo cual ha sido visto en otros estudios chilenos (Chávez \& Verga$\mathrm{ra}, 2017$ ). La separación de los padres y las nuevas configuraciones familiares requieren una resignificación de la identidad personal, al tener que integrar diferentes relaciones que implican reorganizaciones de la vida cotidiana, que podrían ser difíciles de acomodar.

En niños y adolescentes se aprecia la necesidad de integrar roles que le den continuidad al sí mismo, a través de la búsqueda activa de actividades en el presente; en estas reconocen y descubren intereses, motivaciones, deseos y pasiones. Valoran las actividades artísticas y deportivas que entregan bienestar y placer, que les permiten reconocerse y ser reconocidos por otros a través de la acción y proyectarse al futuro. Dicha proyección resulta ser un hallazgo relevante de esta investigación, presentándose desde los 9 años y se da especialmente a través del proyecto académico y profesional.

En la integración con otros, se observan resultados novedosos desde la teoría constructivista evolutiva. Se aprecia que tanto los niños como los adolescentes incorporan a su familia y pares como parte relevante de la propia identidad, lo que da un sentido de afiliación, con la construcción de un nosotros, descubriendo sentidos de vida cooperativos que facilitan la reafirmación personal.

En los niños se observa el reconocimiento desde los otros especialmente a través de la ligazón afectiva y las diferentes actividades realizadas con familiares; mientras que en los adolescentes este reconocimiento se da también a través de la incorporación de valores, pensamientos e ideologías políticas y su acción en diferentes ámbitos, más allá de lo familiar y social. En ellos aparecen deseos de libertad, autonomía y toma de decisiones, necesitando ser agentes en la construcción de su identidad personal. Esto se puede relacionar con la importancia de la integración, no solamente a nivel familiar y escolar, sino también promover la integración a la comunidad de manera más amplia; así, se integran aspectos de la ciudadanía en la configuración de la identidad.

Solo en algunos casos se incluyen otros grupos de pertenencia, lo que nos muestra la necesidad de enfatizar en todos los niveles de acción el sentido de la cooperación social e integración a grupos, más que el individualismo y la competencia. La cooperación lleva a los niños y adolescentes a la práctica de la reciprocidad y a la generosidad en sus relaciones 
sociales; desarrollando el respeto entre las personas como un elemento central de la vida social (Sepúlveda, 2020). Desde el constructivismo, según Piaget (1983), el estado de equilibrio en lo social propicio para el desarrollo humano es el estadio de la cooperación en el cual los individuos, considerándose como iguales, pueden regularse mutuamente y realizar acciones en común.

Como conclusión, podemos señalar que la identidad se comienza a organizar desde la infancia, lo cual es coherente con lo mencionado por otros autores (Beyers \& Luyckx, 2016; Negru-Subtirica et al., 2016; Sepúlveda, 2020). Así mismo, se observan diferencias evolutivas en su construcción desde la infancia a la adolescencia, por lo cual la identidad va cambiando con el desarrollo, integrándose cada vez más elementos a ella, complejizándose su construcción.

En esta línea, dentro de las principales diferencias evolutivas observadas en los diversos rangos etarios (figura 1), podemos apreciar a partir de los resultados que la identidad personal en niños de 6 a 8 años se configura principalmente desde el reconocimiento de sí, destacando los aspectos tangibles y sensoriales; ello refleja la etapa del desarrollo en la que se encuentran, de transición del pensamiento preoperatorio a operatorio concreto (Piaget, 1995). Entre los 9 y 15 años los niños empiezan a incluir más aspectos de la integración de sí mismo e integración con los otros en sus narrativas. Esto puede comprenderse desde aspectos evolutivos, tales como la presencia de un pensamiento concreto y su transición a un pensamiento formal (Piaget, 1995); esto implica el desarrollo de esquemas conceptuales operatorios que persisten en el tiempo, a pesar de los cambios que se producen constantemente en las configuraciones familiares, escolares y sociales.

Entre los 16 y 18 años se observa una reflexión de la historia de vida, integrando aspectos ideológicos, en el ámbito social, religioso y político, y se contextualiza la propia identidad en la historia, tradición y cultura (Bruner, 1991); es central la relación del significado de sí mismo con el otro, lo cual puede dar cuenta del desarrollo del pensamiento operatorio formal (Piaget, 1995). Esto se observa en narrativas con sentidos y significados coherentes y también en la referencia a estados internos, especialmente experiencias emocionales y compromisos ideológicos.

Estos resultados son coherentes con el modelo de desarrollo epigenético de Erikson $(1993,1994)$, que plantea que, si bien la adolescencia es la etapa central de construcción de identidad, esta comienza su desarrollo previamente. En este contexto, esta investigación contribuye con conocimientos empíricos de la construcción de la identidad personal en niños, sumando a las investigaciones ya existentes en identidad en la adolescencia 
(Klimstra et al., 2010; Meeus, 1996; Zacarés et al., 2009), aportando en la distinción del desarrollo evolutivo de la identidad, especialmente en grupos de adolescentes y preadolescentes en Chile.

Esta investigación presenta algunas limitaciones. La primera es la escasa variabilidad de la muestra, en términos socioeconómicos y solo un establecimiento educacional. Segundo, el estudio se enfoca en un momento particular del desarrollo, lo que sugiere futuros estudios que centren el análisis en lo longitudinal, para conocer cómo se desarrolla el proceso de construcción identitaria. Tercero, es relevante considerar que la escritura de las narrativas podría estar influida por aspectos evolutivos, tales como las capacidades narrativas, el desarrollo del lenguaje y la lectoescritura, las cuales no fueron evaluadas en este estudio. Por último, y dado el contexto actual en temáticas de género y la relevancia de estas en la clínica con niños y adolescentes, el estudio tiene la limitación de no abordar en profundidad este aspecto de la identidad, quedando el desafío de afrontarlo desde esta perspectiva teórica en futuras investigaciones.

Futuros estudios podrían considerar complementar y enriquecer el análisis con otros desarrollos teóricos en identidad, ya que existen investigaciones recientes que analizan la identidad en relación a los planteamientos de Marcia y autores posteriores en su línea de pensamiento (Reese et al., 2017; Žukauskienè et al., 2018). Otra línea de investigación, dados los resultados, es profundizar en la relevancia del contexto sociocultural en la construcción identitaria (Díaz-Barriga et al., 2019); específicamente, en la elección de los proyectos vitales y profesionales que los jóvenes hacen, incorporando diversos contextos culturales, países o niveles socioeconómicos en la investigación, dada la relevancia que esto podría tener en el ámbito de la educación, clínica y promoción de salud en niños y adolescentes.

Las implicancias se relacionan con la promoción del desarrollo de la identidad en niños y adolescentes. Otros estudios han observado la relevancia de la identidad en las relaciones interpersonales, la integración social y el bienestar personal (Crocetti et al., 2017; Juang et al., 2020; Reese et al., 2017). En este sentido, a partir de los resultados del estudio, sería relevante promover el desarrollo de la identidad desde la infancia y no solo concentrar los esfuerzos en la adolescencia, favoreciendo la construcción de la identidad diferenciada e integrada y, por lo tanto, un desarrollo adaptativo en todas las edades, considerando las diferencias evolutivas encontradas en este estudio. Otra implicancia tiene relación con los sistemas sociales (colegio, familia, comunidad), considerando la relevancia del contexto cultural, social y escolar en la definición identitaria (Aguirre-Pastén et al., 
2017; Díaz-Barriga et al., 2019; Ortiz-Piedrahía, 2020), siendo importante organizar sistemas más inclusivos, de modo que cada persona pueda descubrir quién es y quien quiere ser, valorando su unicidad y distinción de otros, sin la expectativa de tener que llegar a alcanzar la perfección (Byung-Chul Han, 2017). En ese sentido, estos resultados, nos presentan el desafío de validar y desarrollar diversas actividades que sean elegidas según los intereses y necesidades de niños y jóvenes, no solo concentrando la valoración en actividades académicas, posiblemente implicando redefinir con mayor flexibilidad los contenidos que se integran en la escuela, los cuales favorezcan los procesos de diferenciación e integración en la construcción de su identidad.

Una última implicancia se relaciona con la relevancia con que aparecen las nuevas organizaciones y configuraciones familiares y cómo ellas se integran en la identidad de niños y adolescentes. Se destaca la necesidad de reconocer a nivel social la diversidad en la configuración y los significados asociados a la familia; también el rol que el psicólogo representa, de acuerdo a los resultados, en favorecer la integración de estas diferentes vivencias en la identidad, facilitando nuevos equilibrios.

\section{Referencias}

Aguirre-Pastén, B., Gajardo-Tobar, A., \& Muñoz-Madrid, L. (2017). Construcción de identidad de la niñez en contextos de ruralidad en la comuna de Concepción, Chile. Revista Latinoamericana de Ciencias Sociales, Niñez y Juventud, 15(2), 893-911.

Asociación de Investigadores de Mercado. (2015). Estudio completo sobre el nuevo modelo de grupos socioeconómicos. https://www.aimchile.cl/gse-de-chile/

Beyers, W., \& Luyckx, K. (2016). Ruminative exploration and reconsideration of commitment as risk factors for suboptimal identity development in adolescence and emerging adulthood. Journal of Adolescence, 47, 169-178. https://doi.org/f8b5hr

Bonilla-García, M., \& López-Suárez, A. (2016). Ejemplificación del proceso metodológico de la teoría fundamentada. Cinta Moebio, (57), 205-315. https://doi.org/gdvh4n

Braun, V., \& Clarke, V. (2006). Using thematic analysis in psychology. Qualitative Research in Psychology, 3(2), 77-101. https://doi.org/10.1191/1478088706qpo6zoa

Brown, P., Capella, C., \& Antivilo, A. (2014). Propiedades psicométricas de la versión para padres del Strenghts and difficulties questionnaire. Revista de Psicología Universidad de Chile, 23(2), 28-44. https://doi.org/10.5354/0719-0581.2014.36146

Bruner, J. (1991). Actos de significado: más allá de la revolución cognitiva. Alianza. 
Byung-Chul, Han. (2017). La expulsión de los distinto. Herder.

Capella, C. (2011). Hacia narrativas de superación: el desafío para la psicoterapia con adolescentes de integrar la experiencia de agresión sexual a la identidad personal [Tesis doctoral, Universidad de Chile]. Repositorio Universidad de Chile. https://bit.ly/3ybRTG4

Capella, C. (2013). Una propuesta para el estudio de la identidad con aportes del análisis narrativo. Psicoperspectivas, $12(2), 117-128$.

Capella, C. (2017). Disequilibrium and loss of narrative coherence in identity construction: A Piagetian perspective on trauma in adolescent victims of sexual abuse, Journal of Constructivist Psychology, 30(4), 323-338. https://doi.org/10.1080/10720537.2016.1227737

Catalán, P. (2004). Evaluación del estado de desarrollo del sí mismo, desde una perspectiva constructivista evolutiva, en adolescentes embarazadas [Tesis de maestría no publicada]. Universidad de Chile.

Cifuentes-Muñoz, A., \& Rojas-Jara, C. (2018). La fotografía como medio narrativo para la co-construcción de identidades alternativas en contexto de abuso de drogas. Revista Latinoamericana de Ciencias Sociales, Niñez y Juventud, 16(1), 89-10o. https://doi.org/gcgw

Cornejo, M., \& Salas, N. (2011). Rigor y calidad metodológicos: un reto a la investigación social cualitativa. Psicoperspectivas, 10(2), 12-34. https://doi.org/cs5s

Crafter, S., de Abreu, G., Cline, T., \& O’Dell, L. (2015). Using vignette methodology as a tool for exploring cultural identity positions of language brokers. Journal of Constructivist Psychology, 28(1), 83-96. https://doi.org/10.1080/10720537.2014.923354

Crocetti, E., Branje, S., Rubini, M., Koot, H., \& Meeus, W. (2017). Identity processes and parent-child and sibling relationships in adolescence: A five-wave multi-informant longitudinal study. Child Development, 88(1), 210-228. https://doi.org/10.1111/cdev.12547

Crockett, M. (2013). Imagen de sí mismo en niños con trastorno por déficit de atención e hiperactividad [Tesis de pregrado, Universidad de Chile]. Repositorio Universidad de Chile. http://repositorio.uchile.cl/handle/2250/130007

Chávez, P., \& Vergara, A. (2017). Ser niño y niña en el Chile de hoy: la perspectiva de sus protagonistas acerca de la infancia, la adultez y las relaciones entre padres e hijos. Ceibo.

De la Harpe, M. (2005). Desarrollo de la organización del sí mismo desde una perspectiva constructivista evolutiva en niños y adolescentes de 8 a 20 años [Tesis de maestría no publicada]. Universidad de Chile.

Díaz-Barriga, F., Vázquez-Negrete, V. I., \& Díaz-David, A. (2019). Sentido de la experiencia escolar en estudiantes de secundaria en situación de vulnerabilidad. Revista Latinoamericana de Ciencias Sociales, Niñez y Juventud, 17(1), 237-252. https://dx.doi.org/ 10.11600/1692715X.17114 
Ergün, N. (2020). Identity development: Narrative identity and intergenerational narrative identity. Psikiyatride Guncel Yaklasimlar-Current Approaches in Psychiatry, 12(4), 455-475. https://doi.org/10.18863/pgy.676439

Erikson, E. (1993). Infancia y sociedad. Horme; SAE.

Erikson, E. (1994). Identity, youth and crisis. W. W. Norton.

Foelsch, P., Schlüter-Müller, S., Odom, A., Arena, H., Borzutzky, A., \& Schmeck, K. (2015). Tratamiento para la identidad del adolescente (AIT). Manual Moderno.

González, N. (2011). Psicopatología evolutiva desde la perspectiva constructivista Piagetana en adolescentes de 14 y 15 años [Tesis de maestría no publicada). Universidad de Chile.

Goodman, R. (1997). The strengths and difficulties questionnaire: A research note. Journal of Child Psychology and Psychiatry, 38(5), 581-586. https://doi.org/b6bmr3

Goth, K., Foelsch, P., Schlüter-Müller, S., Birkhölzer, M., Jung, E., Pick, O., \& Schmeck, K. (2012). Assessment of identity development and identity diffusion in adolescenceTheoretical basis and psychometric properties of the self-report questionnaire AIDA. Child and Adolescent Psychiatry and Mental Health, 6. https://doi.org/ghfbwp

Guidano, V. (1994). El sí mismo en proceso. Paidós.

Hernández, B. (2012). Construcción de identidad en adolescentes nadadoras de nivel competitivo y su relación con los significados asociados al compromiso deportivo [Tesis de maestría, Universidad de Chile]. Repositorio Universidad de Chile. https://bit.ly/3yaDUAe

Hernández, R., Fernández, C., \& Baptista, M. P. (2006). Metodología de la investigación. McGraw-Hill.

Juang, L., Schachner, M., Pevec, S., \& Moffitt, U. (2020). The Identity Project intervention in Germany: Creating a climate for reflection, connection, and adolescent identity development. New Directions for Child and Adolescent Development, (173), 65-82. https://doi.org/10.1002/cad.20379

Kegan, R. (1994). The evolving self. Harvard University Press.

Kirk, S., \& Hinton, D. (2019). «I'm not what I used to be»: A qualitative study exploring how young people experience being diagnosed with a chronic illness. Child Care Health, 45(2), 216-226. https://doi.org/10.1111/cch.12638

Klimstra, T., Hale, W., Raaijmakers, Q., Branje, S., \& Meeus, W. (2010). Identity formation in adolescence: change or stability? Journal of Youth and Adolescence, 39(2), 150-162. https://doi.org/10.1007/s10964-009-9401-4

Klym, M., \& Cieciuch, J. (2015). The early identity exploration scale-a measure of initial exploration in breadth during early adolescence. Frontiers in Psychology, 6(533), 1-8. https://doi.org/10.3389/fpsyg.2015.00533 
Mansilla, M. A., \& Piñones-Rivera, C. (2017). Lo moreno es bello: componentes identitarios de las mujeres jóvenes evangélicas aymaras. Revista Latinoamericana de Ciencias Sociales, Niñez y Juventud, 15(2), 1005-1019.

Martin, A. (2000). Evaluación del estado de desarrollo del sí mismo, desde una perspectiva constructivista evolutiva, en jóvenes consumidores abusivos de marihuana y alcohol, a través de sus narrativas [Tesis de maestría, Universidad de Chile]. Repositorio Universidad de Chile. http://repositorio.uchile.cl/handle/2250/136216

Meeus, W. (1996). Studies on identity development in adolescence: An overview of research and some new data. Journal of Youth and Adolescence, 25, 569-598. https:// doi.org/10.1007/bfo1537355

Murphy, A. (2018). Charting the emergence of national identity in children in Wales. Children \& Society, 32(4), 301-313. https://doi.org/10.1111/chso.12252

Negru-Subtirica, O., Pop, E. I., Luyckx, K., Dezutter, J., \& Steger, M. F. (2016). The meaningful identity: A longitudinal look at the interplay between identity and meaning in life in adolescence. Developmental Psychology, 52(11), 1926-1936. https://doi.org/fgbfox

Oron, J. V., \& Echarte, L. E. (2017). Consideraciones sobre el intervalo de vulnerabilidad de la adolescencia. Cuadernos de Bioética, XXVIII(1), 13-27.

Ortiz-Piedrahíta, V. (2020). Subjetivación de la blanquitud por jóvenes universitarios: un estudio comparativo. Revista Latinoamericana de Ciencias Sociales, Niñez y Juventud, 18(1), 1-24. http://dx.doi.org/10.11600/1692715x.18109

Papalia, D., Wendkos, S., \& Duskin, R. (2010). Desarrollo humano. McGraw-Hill.

Piaget, J. (1983). El criterio moral en el niño. Fontanella.

Piaget, J. (1995). Seis estudios de psicología. Labor.

Procaccia, R., Veronese, G., \& Castiglioni, M. (2014). Self-characterization and attachment: A creative method of investigating children's construing. Journal of Constructivist Psychology, 27(3), 174-193. https://doi.org/10.1080/10720537.2014.904701

Ramírez, C., \& Salas, I. (2013). Construcción de identidad en adolescentes con intento de suicidio [Tesis de pregrado, Universidad de Chile]. Repositorio Universidad de Chile. http:// repositorio.uchile.cl/handle/2250/130701

Reese, E., Myftari, E., McAnally, H., Chen, Y., Neha, T., Wang, Q., Jack, F., \& Robertson, S. (2017). Telling the tale and living well: Adolescent narrative identity, personality traits, and well-being across cultures. Child Development, 88(2), 612-628. https:// doi.org/10.1111/cdev.12618 
Rivas-Drake, D., Umaña-Taylor, A., Schaefer, D., \& Medina, M. (2017). Ethnic-racial identity and friendships in early adolescence. Child Development, 88(3), 710-724. https://doi.org/10.1111/cdev.12790

Rodríguez, G., Gil, J., \& García, E. (1999). Metodología de la investigación cualitativa. Aljibe.

Rossel, J. I., \& Torrejón, D. (2019). Identidad personal en niñas y niños de 6 a 12 años del colegio rural Las Palmas de Llay-Llay: dimensiones de la identidad [Tesis de pregrado no publicada]. Universidad de Chile.

Sepúlveda, M. G. (2020). Psicoterapia constructivista evolutiva con niños y adolescentes. Mediterráneo.

Tatlow-Golden, M., \& Guerin, S. (2010). «My favourite things to do» and «my favourite people»: Exploring salient aspects of children's self-concept. Childhood, $17(4), 545-562$. https://doi.org/10.1177/0907568210364667

Thomas, L. (2015). Fostering resilience: Exploring former foster children's narratives [Tesis doctoral, University of Iowa]. Iowa Research Online. http://ir.uiowa.edu/etd/1775

Turban, J., \& Ehrensaft, D. (2018). Research review: Gender identity in youth: Treatment paradigms and controversies. Journal of Child Psychology and Psychiatry, 59(12), 1228-1243. https://doi.org/10.1111/jcpp.12833

Valdés, N., Hernández, C., Goth, K., Quevedo, Y., \& Borzutzky, A. (2019). Adaptación y validación de la versión chilena del cuestionario para evaluar el desarrollo de la identidad en adolescentes (AIDA). Revista Argentina de Clínica Psicológica, XXVIII(5), 610-623.

Yáñez, C. (2014). Construcción de identidad personal en niños y niñas de 5 a 9 años pertenecientes al pueblo originario Aymara, residente en la comuna de Putre [Tesis de maestría, Universidad de Chile]. Repositorio Universidad de Chile. https://bit.ly/3bqTHkF Zacarés, J., Iborra, A., Tomás, J., \& Serra, E. (2009). El desarrollo de la identidad en la adolescencia y adultez emergente: una comparación de la identidad global frente a la identidad en dominios específicos. Anales de Psicología, 2(25), 316-329.

Žukauskienè, R., Truskauskaitè-Kunevičienėa, I., Kaniušonytėa, G., \& Crocetti, E. (2018). How do Lithuanian adolescents address identity questions? A four-wave longitudinal study on change and stability in identity styles. European Journal of Developmental Psychology, 15(1), 41-6o, https://doi.org/10.1080/17405629.2017.1285762 